\title{
The effect of zoledronic acid on growth plates and high turnover bones
}

\author{
Erdogan $\mathrm{M}^{1}$, Bereket $\mathrm{C}^{2}$, Ozkan $\mathrm{N}^{2}$, Alici $\mathrm{O}^{3}$, Sener $\mathrm{I}^{2}$, Desteli EE ${ }^{4}$, Ilkaya $\mathrm{F}^{5}$ \\ Department of Orthopedic, Faculty of Medicine, Ondokuz Mayis University, Samsun, Turkey. \\ erendesteli@yahoo.co.uk
}

\begin{abstract}
Objectives: Bisphosphonates have preventive effect on bone resorption caused by osteoclasts. We aimed to investigate the histopathological effects of zoledronic acid (ZA) on the jaw and long bones and growth plates of rats.

Methods: Thirty-six 12 week-old female Sprague-Dawley rats were divided into the control $(C, n=18)$ and ZA groups $(Z, n=18)$. $Z$ group animals were administered $0.1 \mathrm{mg} / \mathrm{kg}$ saline-diluted $Z A$ intraperitoneally three times per week for 8 weeks. $C$ group animals were administered the same amount of saline simultaneously. At the end of 11th week, half the subjects from either the control group (C1) and ZA group (Z1) were sacrificed. At the end of 14 th week, the remaining half from both groups were also sacrificed (C2 and $Z 2$ ). In all animals, no dental procedures were performed; the posterior and anterior mandible and the knee joint including distal femur and proximal tibia were histopathologically investigated.

Results: Histological examination revealed that inflammation and necrosis were limited to the posterior mandible of the $\mathrm{Z} 1$ and $\mathrm{Z} 2$ groups, while the anterior mandible and knee joint including distal femur and proximal tibia remained unaffected however the development of the growth plate of the proximal tibia was found to be arrested in animals of the Z1 and Z2groups.

Conclusion: Due to it is inhibitory effect over growth plate and inflammatory and necrotic effect over high turnover bones, zoledronic acid should be administered cautiously, especially in pediatric patients who are still in their growth and development stages (Fig. 6, Ref. 34). Text in PDF www.elis.sk.

Key words: inflammation, epiphysis, bisphosphonates.
\end{abstract}

Bisphosphonates (BPs) are synthetic analogues of pyrophosphate and they have a preventive effect on bone resorption caused by osteoclasts (1). Thus, they elevate bone mineral density and reduce the risk of bone fracture. Due to these effects, BPs are widely used in clinical practice, extending from treatment of metabolic bone diseases and osteoporosis to treatment of primary and metastatic bone tumors (2)

BPs, due to their different structural features, may represent miscellaneous clinical and biological efficacy and they are divided into two groups. The first group involves the non-aminobisphosphonates, i.e., BPs that do not include nitrogen such as clodronate and etidronate. The second group involves the aminobisphosphonates, i.e., BPs that include nitrogen such as zoledronate, pamidronate, ibandronate, alendronate, and risedronate. Zoledronic acid (ZA) belongs to the third generation BPs. BPs have a high affinity to calcium so target to bone tissue. Aminobisphosphonates have higher efficacy than do non-aminobisphosphonates, thus they

${ }^{1}$ Department of Orthopedic, Faculty of Medicine, Ondokuz Mayis University, Samsun, Turkey, ${ }^{2}$ Department of Oral and Maxillofacial Surgery, Faculty of Dentistry, Ondokuz Mayis University, Samsun, Turkey, ${ }^{3}$ Samsun Education and Research Hospital, Samsun, Turkey, ${ }^{4}$ Uskudar State Hospital, Istanbul, Turkey, and ${ }^{5}$ Department of Pharmacology, Faculty of Medicine, Ondokuz Mayis University, Samsun, Turkey

Address for correspondence: E.E. Desteli, MD, Uskudar State Hospital, Istanbul, Turkey.

Phone: +90.532 .2267153$ are more frequently used (3). Zoledronic acid (ZA) is the most efficient BP and is thus most commonly used in clinical practice (4). However, some unexpected side effects of BPs have been reported in clinical practice, particularly for zoledronic acid. One of the most common side effects is osteonecrosis found only in the jawbones (BRONJ, Bifosfonat-Related-Osteonecrosis of the Jaws $(5,6)$. Although several case reports have suggested that factors such as dental extraction, trauma, and periodontal diseases may act as cofactors in the pathogenesis of BRONJ in patients receiving BPs, this finding is yet to be confirmed $(7,8)$.

Few experimental and clinical trials have aimed to determine the factors influencing the effects of BPs on jaws and other longer bones. Nevertheless, it has been suggested that bones of the skull and those of the appendicular skeleton give different responses to BPs since they have developed from distinct cell lineages during embryological development (9).

In recent years, the method of distraction osteogenesisis being used in the clinical treatment of many diseases (9) and BPs have been reported to elevate the strength and quality of callus formed due to distraction (10). Some studies have also reported that BPs damage the growth plate and lead to development of shortness by preventing normal bone elongation. However, studies focusing on this topic are limited in number and animal model studies are required (11).

Therefore, we aimed to investigate the effects of ZA, one of the most effective and commonly used BP in clinical practice, on 
the growth plate of developing bones and in the development of BRONJ in a rat model, without taking into consideration the cofactors such as dental extraction, periodontal diseases, and trauma.

\section{Methods}

The protocol for this study was approved by the Animal Care and Ethics Committee of our institution. We used thirty-six 12-week-old female Sprague-Dawley rats weighing 220-300 g. All of the animals were housed in separate cages, and were provided with a nutritive diet with free access to food and water. The experiments were performed between 9 am and $5 \mathrm{pm}$ to avoid the effect of diurnal variation. The rats were maintained in this environment for 2 weeks for acclimatization, and were then randomly divided into two groups: control group $(C, n=18)$ and ZA group $(\mathrm{Z}, \mathrm{n}=18)$. The animals of the $\mathrm{Z}$ group were administered $0.1 \mathrm{mg} /$ $\mathrm{kg}$ saline-diluted ZA (Zometa; Novartis, Istanbul, Turkey) intraperitoneally three times per week for 8 weeks. The animals of the $\mathrm{C}$ group were administered the same amount of saline simultaneously. The dose and time of administration of ZA was determined
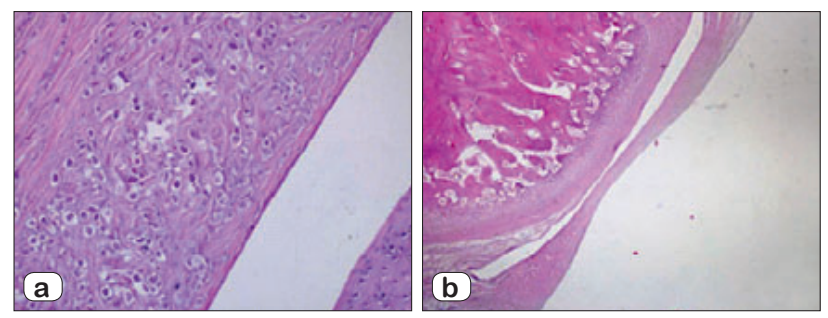

Fig. 1a and b. Mandibular joint surface of an animal from the control group. No inflammation or necrosis is seen in the cartilage tissue (a: H\&E, ×40; b: H\&E, ×200).

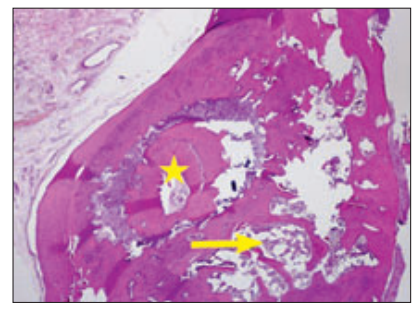

Fig. 2. A mixed-type inflammatory cell infiltration progressing towards the dental root (star) and the alveolar bone (arrow) in the posterior mandible $(\mathrm{H} \& \mathrm{E}, \times 40)$.
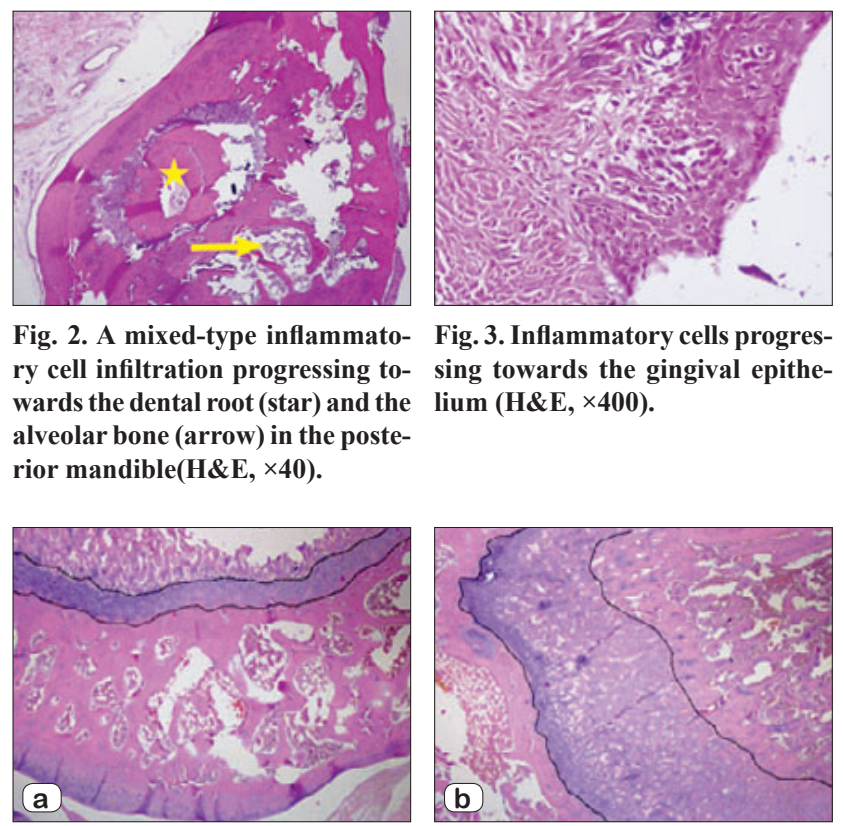

Fig. 3. Inflammatory cells progressing towards the gingival epithelium (H\&E, ×400).

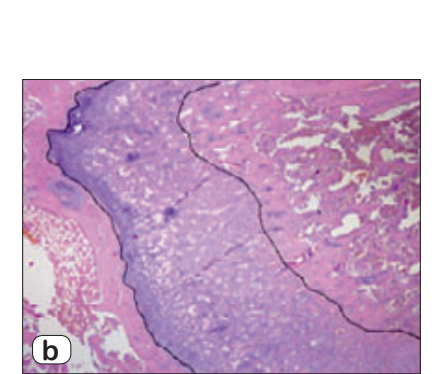

according to previous studies. All administrations were performed by the same surgeon. At the end of the 11th week, i.e., three weeks after the end of treatment period, half the rats from both the control group $(\mathrm{C} 1)$ and the ZA (Z1) group were sacrificed by injecting high-dose pentobarbital (Pental; IE Ulagay, Istanbul, Turkey) and at the end of 14th week, the remaining rats in each group, the control group (C2) and ZA (Z2), were sacrificed using the same procedure. In all animals, anesthesia was induced by injecting a mixture of ketamine/xylazine intraperitoneally. After sacrificing the animals, a single surgeon obtained specimens from their jawbones and knee joints, including the distal femur and the proximal tibia. Obtained specimens were fixed with $10 \%$ buffered neutral formalin solution within the next 24 hours. Following fixation, the obtained bone specimens were decalcified using $25 \%$ formic acid solution. The specimens were then subjected to routine follow-up after being embedded in paraffin blocks. Four- $\mu$ m-thick sections of these blocks were stained using hematoxyllin-eosin (H\&E). A complete histopathological evaluation was performed by a single pathologist who was blinded to the groups. Changes in the growth plate of the proximal tibia were also concurrently examined. The density of inflammation was evaluated semi quantitatively. The length of the growth plates were measured using an Olympus DP72 camera (Tokyo, Japan).

\section{Results}

Histopathological investigation involved examination of the specimens obtained from the jaw bone and the knee joint. There was no histopathologically detected inflammation or necrosis in the jaw bones and knee joints in control groups (C1 and $\mathrm{C} 2)$ (Figs $1 \mathrm{a}$ and $1 \mathrm{~b})$.
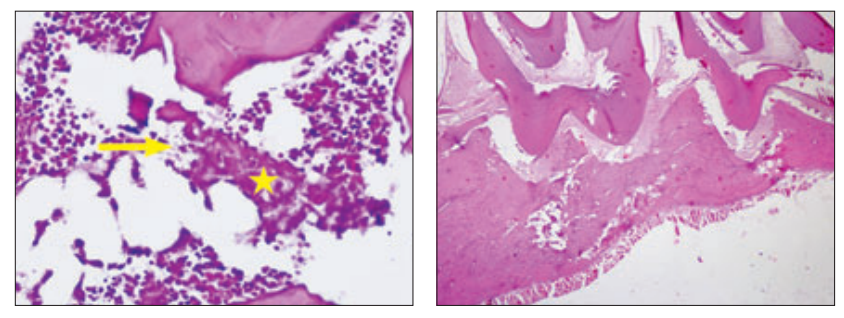

Fig. 4. Necrotic bone spicules in the Fig. 5. Absence of inflammation or alveolar bone (star), mixed-type in- necrosis at the level of the anterior flammation, and coccoid-type bac- mandible $(\mathrm{H} \& \mathrm{E}, \times 40)$. teria $(\mathrm{H} \& \mathrm{E}, \times 400)$.
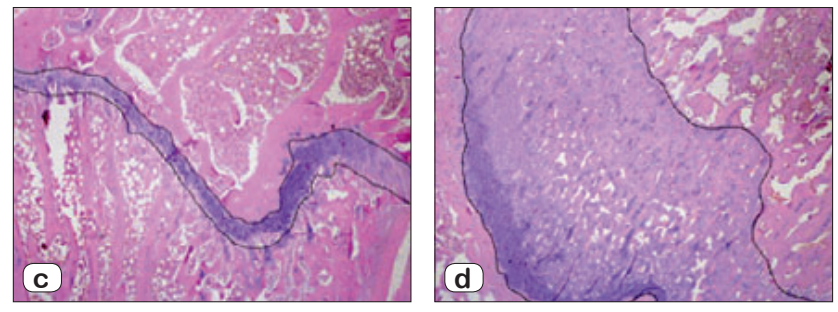

Fig. 6. Difference between the control and study groups with respect to thickness of the growth plates of the proximal tibia. The margins of the growth plates are black (a:C1, 11th week;b:Z1, 11thweek; c:C2, 14th week; d:Z2, 14th week; H\&E, ×40). 
In $\mathrm{Z}$ group ( $\mathrm{Z} 1$ and $\mathrm{Z} 2$ ) rats, inflammation was observed at the level of the gingival epithelium and along the alveolar bone, resulting in a mixed type of inflammatory cell infiltration (Figs 2 and 3). However, we observed that the inflammation was more pronounced in animals from the Z2 group. We also simultaneously encountered mucosal ulcerations in the jaw bones of animals in the Z1 and Z2 groups. These ulcerations appeared to be more in the Z2 group. Necrosis was also detected in histiological examination of posterior mandibula sections in animals of the $\mathrm{Z} 1$ and Z2 groups (Fig. 4).

No inflammation or necrosis was detected at the level of the anterior mandible in animals of both study and control groups (Fig. 5) similarly, in all rats, examination of the specimens obtained from the knee joint revealed no histopathological finding with respect to inflammation or necrosis. However, histopathological investigation of the growth plates in the regions of the proximal tibia showed some differences between the control and study group rats. A discontinuation was found in development of the growth plate of the proximal tibia in the animals of the Z group (Z1 and Z2) (Figs $6 a-d)$. This discontinuation was more pronounced in animals of the $\mathrm{Z} 2$ group. Histopathological examination revealed that, alignement of chondrocytes in physis was significantly disturbed in ZA groups (1-2). Height of physis was found to be decreased in ZA groups. Consequently, in the proximal tibia, the thickness of the growth plates of the control and study groups were found to be different.

\section{Discussion}

Although the mechanisms of action of BPs on bone metabolism are not yet clear, the data obtained from clinical studies have demonstrated the presence of an association between BPs and osteonecrosis (12). BPs show efficacy on bones when administered in physiological dosages. The BPs that include nitrogen inhibit the action of osteoclasts by blocking farnesyldiphosphate synthase $(13,14)$. Although their half-life range between 30 minutes and 2 hours within circulation, they can remain in the bones for up to 10 years depending on the skeletal turnover time (13).

Anti-angiogenic effects of BPs are considered to be responsible for the pathophysiology of BRONJ (15). This effect is induced by decreasing the levels of vascular endothelial factor. Thus, BPs reduce the flow and amount of blood circulation within bone tissues (16).

It is not yet known why BP-induced osteonecrosis is observed only in jawbones. Due to the high bone remodeling rate on the surfaces of periodontal and alveolar bones located in the jaw, it has been suggested that high absorption and accumulation of BPs may be responsible for the pathogenesis of the osteonecrosis in jaw bones (17). It has been demonstrated that accumulated BPs have a toxic effect on the oral epithelium besides exerting an osteonecrotic effect. This toxic effect has been reported to cause an infection in the bone structure and facilitate the formation of osteomyelitis $(18,19)$. In our study, we have observed that osteonecrosis developed only in the jaw bones of animals treated with BPs and that bone structures of the lower extremities were not affected. At the same time, we observed inflammation at the level of the gingival epithelium and along the alveolar bone; this effect was more pronounced in animals of group Z2. In one previous study, mucosal chronic ulceration developed due to soft tissue injury caused by BPs was reported to be the most important factor with respect to the formation of BRONJ (19). In our study, these mucosal ulcerations in jaw bones of rats were detected in both groups $\mathrm{Z1}$ and $\mathrm{Z} 2$ additionally we found this effect to be more prominent in the rats of group $\mathrm{Z} 2$.

Badros et al (20) and Boonyapakorn et al (21) have reported that inflammation and necrosis scores were much higher for the posterior mandible than for the anterior mandible. The underlying reason for this was found to be higher rates of bone turnover and bone remodeling for the posterior mandible than those for the anterior mandible. Similarly, inflammation and necrosis was found only in the posterior mandible of the animals in our study; no histopathological change was observed in the anterior mandible.

Many studies have focused on the association between the duration of BP administration and the development of BRONJ. Allen and Burr administered high-dose ZA to healthy dogs for 3 years and observed necrosis in their jaw bones (22). In our study, necrosis was detected in the jaw bones of the animals in the study group (Z1 and Z2) following administration of ZA for a relatively short duration.

The necrosis and inflammation scores of the affected jaw bones in the study group animals were found to be different from each other, according to previous studies, this disparity could be due to genetic polymorphism (23) .

Smith et al (24) detected a $3 \%$ reduction in the length of extremities of animals treated with zoledronic acid, and they interpreted this as a result of impaired cellular organization in the growth plate and delayed removal of cartilaginous matrix. Camacho et al (25) reported that BPs may reduce the longitudinal growth rate of the growth plate and that this effect may vary depending on the dosage and duration of the administered medication and age. BPs have a high tropism to the bone matrix and accumulate in this tissue (26). Nitrogen containing BPs are increasingly involved in skeletal pediatric disorders such as osteogenesis imperfeca, juvenile rheumatoid arthritis or pediatric cancers like osteosarcoma $(27,28,29,30)$. Besides its preventive effect on bone resorption the third generation bisphosphonate zoledronic acid has been shown to display an inhibitory effect on bone metastases of various human cancers. There are some recent studies published on this topic indicating the antitumoral effect of ZA on primary tumors and metastases (31).

In their study Battaglia et al reported that use of zoledronic acid had an inhibitory effect on long bones and caused growth arrest (32). There are only a few studies to histologically prove growth plate disturbance due to an effect of ZA on physeal morphology (33). In their study Pataki et al reported that subcutaneous injection of ZA for ten days did not make any significant changes in growth plate thickness, however they found that a significant decrease occurred in the longitudinal growth rate due to ZA treatment (11). In one of the histopathological studies it was reported that amount of cartilaginous matrix in resorptive zone of growth plate was increased due to effect of ZA on growth plate whereas in our study 
we did not observe any significant difference between control and ZA groups regarding this issue. In our study, bone volume within both resorptive zone of proximal tibia and metaphysis was found significantly more in ZA groups (1-2) compared to control group whereas in a similar previous study of growth plate, bone volume in resorptive zones of both ZA treated and non-treated groups was found to be comparable (33).

We observed a discontinuation in the development of the growth plate of the proximal tibia in all animals of the study groups (Z1 and Z2). This discontinuation was more prominent in group Z2. Consequently, we found growth plate thicknesses of the control and study groups to be different.ZA suppresses matrix metalloproteinase (MMP-9) expression and prevents apoptosis of endothelial cells via vascular endothelial growth factor (VEGF) (34).

We believe that before the clinical use of ZA increases more in pediatric population, the inhibitory effect of this drug on growth plate and its safety should be thoroughly investigated.

As a contribution to literature knowledge, in this study it was found that, one of the most effective BPs in clinical treatment, leads to inflammation and necrosis in the posterior mandible of the jaw bones, but not in other bones of the skeletal system and causes pathological changes in the cellular morphology of the growth plate. In growth plate it delays the removal of cartilaginous matrix leading to discontinuation in the development of the growth plate of the proximal tibia. In our study these histopathological findings were detected following administration of ZA for a relatively short duration when compared to previous studies. Thus, we propose that ZA should be administered cautiously, especially in pediatric patients who are still in their growth and development stages.

\section{References}

1. World Health Organization. Prevention and management of osteoporosis (WHO Technical Report Series 921). http: //whqlibdoc.who.int/trs/ WHO_TRS_921.pdf, last accessed May 21, 2010.

2. Russell RG, Rogers MJ. Bisphosphonates: From the laboratory to the clinic and back again. Bone 1999; 25: 97-106.

3. Van Beek ER, Cohen LH, Leroy IM et al. Diferentiating the mechanisms of antiresorptive action of nitrogen containing bisphosphonates. Bone 2003; 33: 805-811.

4. Bilston LE, Little DG, Smith NC et al. Zoledronic acid improves the mechanical properties of normal and healing bone. Clin Biomech 2002; 17: 716-718.

5. Khosla S, Burr D, Cauley J et al. Bisphosphonate-associated osteonecrosis of the jaw: report of a taskforce of the American Society for Bone and Mineral Research. J Bone Miner Res 2007; 22: 1479-1491.

6. Mehrotra B, Ruggiero S. Bisphosphonate complications including osteonecrosis of the jaw. Hematology Am Soc Hematol Educ Program 2006; 356-360.

7. American Association of Oral and Maxillofacial Surgeons position paper on bisphosphonate-related osteonecrosis of the jaws. J Oral Maxillofac Surg 2007; 65: 369-376.

8. American Association of Oral and Maxillofacial Surgeons position paper on bisphosphonate-related osteonecrosis of the jaws 2009-up- date. Ruggiero SL, Dodson TB, Assael LA et al. American Association of Oral and Maxillofacial Surgeons. J Oral Maxillofac Surg 2009; 67: $2-12$.

9. Eames BF, Helms JA. Conserved molecular program regulating cranial and appendicular skeletogenesis. Dev Dyn 2004; 231: 4-13.

10. Little DG, Cornell MS, Hile MS et al. Effect of Pamidronateon distraction osteogenesis and fixator-related osteoporosis. Injury 2001; 32: 14-20.

11. Pataki A, Muller K, Green JR et al. Effects of short-term treatment with the bisphosphonates zoledronate and pamidronate on rat bone: a comparative histomorphometric study on the cancellous bone formed before, during and aftertreatment. Anat Rec 2007; 249: 458-468.

12. Hoff AO, Toth BB, Altundag $\mathrm{K}$ et al. Frequency and risk factors associated with osteonecrosis of the jaw in cancer patients treated within intravenous bisphosphonates. J Bone Miner Res 2008; 23: 826-836.

13. Marx RE. Modes of action and pharmacokinetics of the bisphosphonate family. In: Oral and intravenous bisphosphonate-induced osteonecrosis of the jaws: history, etiology, prevention and treatment. Hanover Park, IL: Quintessence Publishing 2007: 11-15.

14. Assael LA. The science of bisphosphonate-related osteonecrosis of the jaws: the thin White line. J Oral Maxillofac Surg 2007; 65: 1275-1276.

15. Cheng A, Mavrokokki A, Carter G et al. The dental implications of bisphosphonates and bone disease. Aust Dent J 2005; 50: 4-13.

16. Kapitola J, Zak J. Effect of pamidronate on bone blood flow in oophorectomized rats. Physiol Res 1998; 47: 237-240.

17. Marx RE. Modes of action and pharmacokinetics of the bisphosphonate family. In: Oral and intravenous bisphosphonate-induced osteonecrosis of the jaws: history, etiology, prevention and treatment. Hanover Park, IL: Quintessence Publishing 2007: 15-17.

18. Sonis ST, Watkins BA, Lyng GD et al. Bony changes in the jaws of rats treated with zolendronic acid and dexamethasone before dental extractions mimic bisphosphonate-related osteonecrosis in cancer patients. Oral Oncol 2009; 45: 164-172.

19. Reid IR, Bolland MJ, Grey AB. Is bisphosphonate-associated osteonecrosis of the jaw caused by soft tissue toxicity? Bone2007; 41: 318-320.

20. Badros A, Weikel D, Salama A et al. Osteonecrosis of the jaw in multiple myeloma patients: clinical features and risk factors. J Clin Oncol 2006: 24: 945-952.

21. Boonyapakorn T, Schirmer I, Reichart PA et al. Bisphosphonateinduced osteonecrosis of the jaws: prospective study of 80 patients with multiple myeloma and other malignancies. Oral Oncol 2008; 44: 857-869.

22. Allen MR, Burr DB. Mandible matrix necrosis in beagle dogs after 3 years of daily oral bisphosphonate treatment. J Oral Maxillofac Surg 2008; 66: 987-994.

23. Migliorati CA, Schubert MM, Peterson DE et al. Bisphosphonate-associated osteonecrosis of mandibular and maxillary bone: an emerging oral complication of supportive cancer therapy. Cancer 2005; 104: 83-93.

24. Smith EJ, McEvoy A, Little DG et al. Transient retention of endochondral cartilaginous matrix with bisphosphonate treatment in a longterm rabbit model of distraction osteogenesis. J Bone Miner Res 2004; 19: $1698-1705$.

25. Camacho NP, Raggio CL, Doty SB et al. A controlled study of the effects of alendronate in a growing mouse model of osteogenesis imperfecta. Calcif Tissue Int 2001; 69: 94-101. 
26. Allen MR. Skeletal accumulation of biphophonates: Implications for osteoporosis treatment. Exp Opin Drug Metab Toxicol 2008; 4: 1371-1378.

27. Brown JJ, Zacharin MR. Safety and efficacy of intravenous zoledronic acid in pediatric osteoporosis. J Pediatr Endocrinol Metab 2009; 22: $55-63$.

28. Rauch F, Cornibert S, Cheung M, Glorieux FH. Long-bone changes after pamidronate discontinuation in children and adolescents with osteogenesis imperfect. Bone 2007; 40: 821-827.

29. Zeitlin L, Rauch F, Plotkin H, Glorieux FH. Height and weight devel- opment during four years of therapy with cyclical intraveinous pamidronate in children and adolescents with osteogenesis imperfecta type I, III, and IV. Pediatrics 2003; 111: 1030-1036.

30. Hasegawa K, Inoue M, Seino Y, Morishima T, Tanaka H. Growth of infants with osteogenesis imperfecta treated with bisphosphonate. Pediatr Int 2009; 51: 54-58.
31. Sewing L, Steinberg F, Schmidt H, Göke R. The bisphosphanate zoledronic acid inhibits the growth of HCT-116 colon carcinoma cells and induces tumor cell apoptosis. Apoptosis 2008; 6: 782-789.

32. Battaglia S, Dumoucel S, Chesneau J, Heymann MF, Picarda G, Gouin F, Corradini N, Heymann D, Redini F. Impact of oncopediatric dosing regimen of zoledronic acid on bone growth: preclinical studies and case report of an osteosarcoma pediatric patient. J Bone Miner Res 2011; 26: 2439-2451.

33. Smith EJ, Little DG, Briody NJ, McEvoy A, Smith NC, Eisman JA, Gardiner EM. Transient disturbance in physeal morphologyis associated with long term effects of nitrogen containing bisphosphonates in growing rabbits. J Bone Miner Res 2005; 10: 1734-1741.

34. Corey E, Brown LG, Quinn JE, Poot M, Roudier MP, Higano CS, Vessella RL. Zoledronic Acid exhibits inhibitory effects on osteoblastic and osteolytic metastases of prostate cancer. Clin Cancer Res 2003; 9: 295-306.

Received September 30, 2013. Accepted January 10, 2014. 